\title{
RHINOLOGY
}

\section{Mathematical model for preoperative identification of obstructed nasal subsites}

\section{Modello matematico per l'identificazione preoperatoria dei sotto-siti nasali sede di ostruzione}

\author{
M. GAMERRA ${ }^{1 *}$, E. CANTONE²*, G. SORRENTINO ${ }^{1}$, R. DE LUCA³, M.B. RUSSO ${ }^{4}$, E. DE CORSO5, F. BOSSA \\ A. DE VIVO ${ }^{6}$, M. IENGO ${ }^{2}$ \\ "ENT Unit, "S. Leonardo" Hospital, Castellammare di Stabia, Italy; ${ }^{2}$ Department of Neuroscience, ENT Unit, \\ University of Naples "Federico II", Naples, Italy; ${ }^{3}$ Department of Physic "E. R. Caianiello", University of Salerno, \\ Salerno, Italy; ${ }^{4}$ Department of Engineering Second University of Naples, Italy; ${ }^{5}$ Catholic University of the Sacred \\ Heart, A Gemelli Hospital, Department of Head and Neck Surgery, Rome, Italy; 6 Neuro Diagnostic Center "Bossa", \\ Torre del Greco, Italy \\ * These authors equally contributed to the study and should be considered first authors
}

\section{SUMMARY}

The planning of experimental studies for evaluation of nasal airflow is particularly challenging given the difficulty in obtaining objective measurements in vivo. Although standard rhinomanometry and acoustic rhinometry are the most widely used diagnostic tools for evaluation of nasal airflow, they provide only a global measurement of nasal dynamics, without temporal or spatial details. Furthermore, the numerical simulation of nasal airflow as computational fluid dynamics technology is not validated. Unfortunately, to date, there are no available diagnostic tools to objectively evaluate the geometry of the nasal cavities and to measure nasal resistance and the degree of nasal obstruction, which is of utmost importance for surgical planning. To overcame these limitations, we developed a mathematical model based on Bernoulli's equation, which allows clinicians to obtain, with the use of a particular direct digital manometry, pressure measurements over time to identify which nasal subsite is obstructed. To the best of our knowledge, this is the first study to identify two limiting curves, one below and one above an average representative curve, describing the time dependence of the gauge pressure inside a single nostril. These upper and lower curves enclosed an area into which the airflow pattern of healthy individuals falls. In our opinion, this model may be useful to study each nasal subsite and to objectively evaluate the geometry and resistances of the nasal cavities, particularly in preoperative planning and follow-up.

KEY WORDS: Nose $・$ Manometry $\bullet$ Fluid dynamics $\bullet$ Airflow $\bullet$ Rhinomanometry

\section{RIASSUNTO}

La realizzazione di studi sperimentali per la valutazione dei flussi aerei nasali è particolarmente indaginosa, data la difficoltà di ottenere in vivo un'accurata misurazione degli stessi. Inoltre, sebbene la rinomanometria standard e la rinometria acustica rappresentino $i$ metodi più utilizzati nella pratica clinica, esse forniscono solo una misura globale ed approssimativa dei flussi aerei nasali, senza definirne i particolari temporali o spaziali. Allo stesso modo gli studi sulla fluidodinamica computazionale rappresentano solo una simulazione numerica, ben lontana da quelle che sono le variabili anatomiche e fisiologiche delle cavità nasali. Pertanto, ad oggi, non esistono ancora strumenti diagnostici in grado di misurare oggettivamente la geometria delle cavità nasali, le resistenze ed il grado di ostruzione nei diversi sotto-siti nasali, elemento quest'ultimo fondamentale per una corretta programmazione chirurgica. Allo scopo di superare i limiti della diagnostica standard abbiamo elaborato un modello matematico basato sull'equazione di Bernoulli applicata alle cavità nasali di soggetti sani per lo studio dei gradienti pressori di vari sotto-siti nasali, che sono stati misurati grazie ad un particolare manometro digitale. Il nostro studio, unico in letteratura, ha identificato due curve limite che racchiudono un'area rappresentativa entro cui cadono i livelli "normali" di flusso in corrispondenza del vestibolo nasale. Il modello descritto potrebbe essere utile per studiare tutti i sotto-siti nasali sede di ostruzione ai fini di una corretta programmazione chirurgica e di un valido follow-up postoperatorio.

PAROLE CHIAVE: Naso $・$ Manometria $\bullet$ Fluidodinamica $\bullet$ Flusso aereo $\bullet$ Rinomanometria 


\section{Introduction}

Nasal obstruction is the most common symptom observed in rhinological practice, afflicting millions of people and accounting annually for over $\$ 5.8$ billion in healthcare costs in the USA alone ${ }^{1}$.

Deviated nasal septum is one of the most common causes of nasal obstruction and septoplasty is the third most common surgery performed by otorhinolaryngologists ${ }^{2}$. However, septal deviation may not always be the only cause of nasal obstruction. Indeed, there are many asymptomatic individuals with nasal deviation and many others suffering from nasal obstruction without septal deviation. Thus, despite successful surgical correction, many patients are not satisfied with the outcomes after septoplasty or nasal surgery in general, which might lead to medico-legal issues. Moreover, some investigators have criticised the high number of unnecessary nasal surgical procedures performed each year ${ }^{2}$.

This happens because very often there are other causes responsible for nasal obstruction, such as nasal valve collapse or lateral wall insufficiency, which may alter the physiological dynamics of the nasal airflow.

For instance, in humans the physiological functions of nasal breathing (conditioning, warming and humidifying of inhaled air, smelling, etc.) are closely related to the mechanical properties of fluids ${ }^{3}$. In healthy adults, most of the airflow occurs in the middle meatus, the nasal respiratory rate at rest is about 16 breaths/min and the airflow volume in a single inspiration is about $500 \mathrm{ml}$. The airflow velocity $(\mathrm{V})$ depends on the strength of breath and on the width of a given nasal subsite, so that during a steady inspiration in standard conditions $\mathrm{V}$ is about $2-3 \mathrm{~m} / \mathrm{sec}$ at the nostril level and $12-18 \mathrm{~m} / \mathrm{sec}$ at the nasal valve area level ${ }^{4}$.

Since the nasal valve and vestibular airflow accounts for $52.6 \%-78.3 \%$ of total nasal airway resistance, these sections are considered the main nasal airflow limiting structures ${ }^{4}$. Thus, this anatomical and physiological narrowing at the "entrance" of the functional segment of the nose causes a significant increase in the velocity of the airflow ${ }^{56}$ and represents the accelerator of the inspired air. Therefore, any obstruction at this level results in a significant reduction in nasal airflow.

To summarise, effective nasal breathing requires certain airflow volumes, a pressure drop, temperature variations and the right proportions between laminar flow and turbulent flow regions, in addition to the ability to provide sufficient air to the lungs ${ }^{7}$.

Hence, from a purely anatomical point of view, changes in the architecture of nasal cavities ${ }^{8}$ for pathological disorders, trauma or surgery may alter the nasal resistances and functions and, consequently, the mechanical properties of nasal airflow, which are strongly affected by the geometry of the airflow passage.
Unfortunately, to date, there are no diagnostic tools to objectively evaluate the geometry and mechanical properties of the nasal cavities and to measure nasal resistance and degree of nasal obstruction. Indeed, despite recent advances in the research of nasal diagnostic strategies, the currently available diagnostic tools for the study of nasal airflow and resistance have some limitations and the individual subjective sensations of the patients do not always match objective measurements ${ }^{9}$.

For instance, the most widely used methods to evaluate the nasal airflow, standard rhinomanometry (RNM) and acoustic rhinometry (AR), can evaluate changes in overall nasal airflow and resistance, and measure cross-sectional areas in nasal cavity. However, due to the structural and physiological complexity of human nose, these techniques are not able to show sufficient details of dynamic airflow through the nasal cavity to fully evaluate many intranasal conditions and anatomical variations, whereas 4-phase rhinomanometry (4PR) might provide supplementary information; nonetheless, all open technical and mathematical inconsistencies conjoint with this technique have been clarified ${ }^{910}$.

Furthermore, the numerical simulation of nasal airflow ${ }^{10} 11$ as computational fluid dynamics (CFD) technology, whose data are not yet validated, requires such a burden of time and resources that it cannot be routinely used ${ }^{12}$.

For all these reasons, the treatment of nasal obstruction in daily clinical practice of otolaryngologists represents a challenge for researchers in constantly searching for reliable objective tests to quantify nasal obstruction.

To overcome these limitations and to objectively measure nasal resistances, the degree and, primarily, the subsite of nasal obstruction, we developed a mathematical model based on Bernoulli's equation applied to clinical practice with the help of the digital DDM-MG1 manometer Endoflower (Gamerra patented, 2007) ${ }^{6}$.

\section{Materials and methods}

The study was performed in accordance with the principles of the 1983 Declaration of Helsinki and was approved by the local board of medical ethics. Written informed consent was obtained from all participants prior to the inclusion in the study. Forty Caucasian subjects, non-smokers suffering from cephalea and without lung disease or nasal obstruction, underwent nasal endoscopy and computed tomography (CT) scan, previously planned for the cephalea, to exclude anatomic variants or sinonasal diseases. The sample was homogeneous for age, sex and body weight ( $20 \mathrm{M} 20 \mathrm{~F}$; mean age $42.64 \pm 13.1$; BMI $\left.22.9 \pm 2.1 \mathrm{~kg} / \mathrm{m}^{2}\right)$.

To carry out pressure measurements, we used a DDMMG1 manometer (Gamerra patented, 2007) ${ }^{6}$. Technical characteristics of the digital manometer were as follows: "Auto Zero" adjustable with respect of the atmospheric 
pressure; range of measurement $=0+/-100$ mbar; overrange pressure: max 300 mbar, resolution: 0.1 mbar (between 0 and 70 mbar), 0,2 mbar (between 70 and 100 mbar); accuracy at $23^{\circ} \mathrm{C}$ : $+/-0.5 \%$ mbar (between 0 and 70 mbar); +/- $1 \%$ mbar (between 70 and 100 mbar); temperature range: $0^{\circ}$ to $+40^{\circ} \mathrm{C}$; not condensing humidity: 10 to $90 \%$ relative humidity.

Under endoscopic guidance we placed the probe of the manometer in the nasal vestibulum corresponding to the CT imaging of the same subsite. Furthermore, to confirm the exact correspondence between the nasal point and the CT scan we chose a point easily detectable corresponding to the dome, part of the vestibulum.

Subjects were asked to slowly and deeply breathe through the nose for 4 seconds, while the nasal section was checked by video-endoscopy for the entire duration of the examination. The manometer was connected to a computer with software for visualisation and recording of pressure values. Images were acquired with an 8-slice CT scanner (LightSpeed, GE Medical Systems, Milwaukee). The volume datasets were processed by a workstation equipped with OsiriX MD software (release 7.0.3, Pixmeo sarl, Bernex, Switzerland) and axial images were reconstructed in coronal and sagittal planes using the MPR (Multi Planar Reconstruction) protocol integrated in the software. The perimeter of air sections was outlined with "pencil" function to obtain the geometric area measurement in $\mathrm{cm}^{2}$.

To estimate the airflow rate through a defined nasal section, we developed software depending on the mathematical model, based on an integral equation, concerning the minimum and maximum value of the area delimited by the two curves in Figure 1, carrying out the volume of the nasal inhaled air for each inhalation.

Assuming the validity of Bernoulli's equation, we can calculate the gauge pressure in the vestibulum by considering one central point $\mathrm{N}$ within this duct. In this expression, we denote the pressure in $\mathrm{N}$ as $p_{N}$. By considering a second point $\mathrm{O}$ at the entrance of the nasal valve and by taking the pressure in $\mathrm{O}$ equal to the atmospheric pressure $p_{a}$, we can write:

$$
p_{N}+\frac{1}{2} \rho V_{N}^{2}=p_{a}
$$

where $r$ is the density of air and $V_{N}$ is the flow velocity in the central part of one section of the nostril, assumed to have circular shape with area $S_{N}$ for simplicity. By taking a parabolic profile as the one shown in Figure 2, we can write:

$$
V(r, t)=V_{N}(t)\left\lceil 1-\left(\frac{r}{R}\right)^{2}\right\rceil
$$

where $r$ is radial distance from point $\mathrm{N}, \mathrm{t}$ is the time measured from the beginning of inhalation, and $\mathrm{R}$ is the radius of the circular section of area $S_{N}$ (Fig. 2).

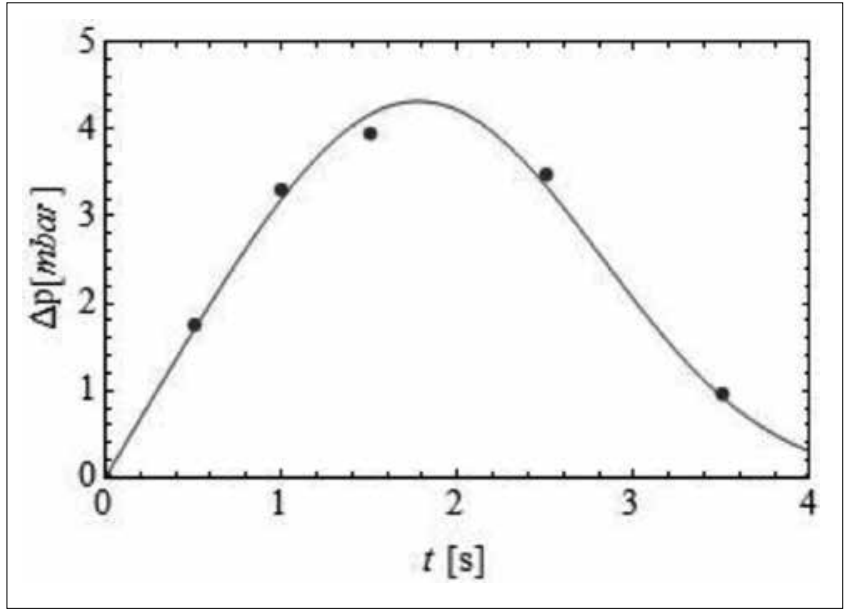

Fig. 1. Minimum and maximum value of the area delimited by the two curves into which the air respiration patterns of healthy people fall.

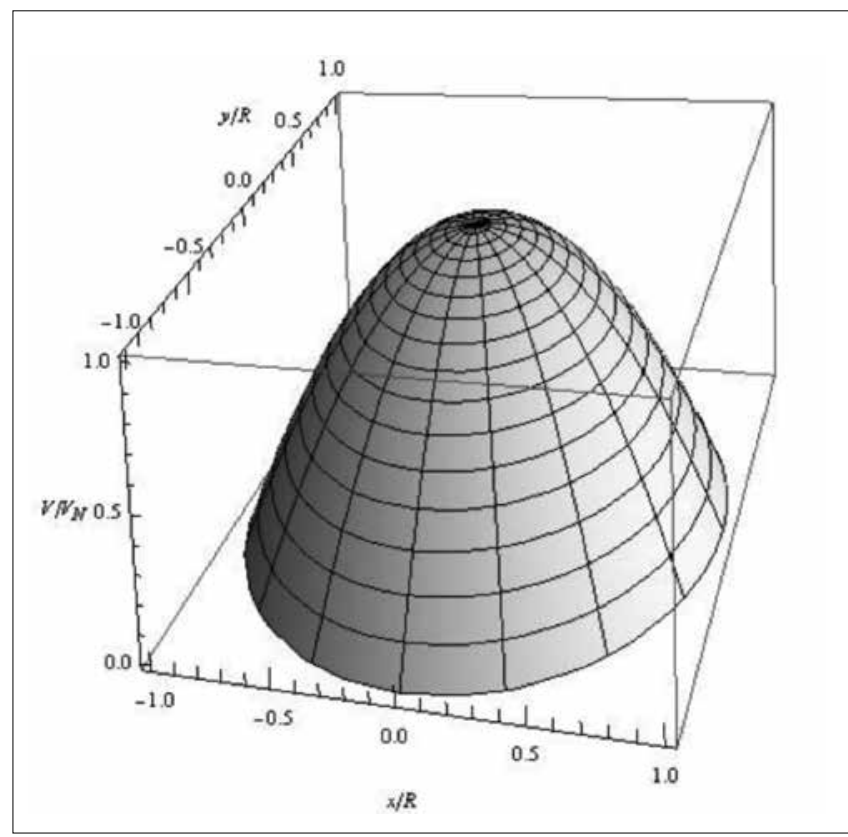

Fig. 2. Parabolic velocity profile of air flowing within a circular duct of radius $R$.

Notice that in Eq. (1) the velocity profile $V(r, t)$ is taken to depend on time through the time dependence of the velocity $V_{N}$. Let us now calculate the flow rate through $S_{N}$, so that we write:

$$
q(t)=\int_{S_{N}} V(r, t) \mathrm{d} S
$$

where $\mathrm{d} S=2 \pi r \mathrm{~d} r$ is the area of an elementary ring of radius $\mathrm{r}$ and thickness within $S_{N}$. By performing the integral, we found:

$$
q(t)=\frac{S_{N}}{2} V_{N}(t)
$$


The $K_{1}=\int_{0}^{\mathrm{T}} q(t) \mathrm{d} t=\frac{S_{N}}{2} \int_{0}^{\mathrm{T}} V_{N}(t) \mathrm{d} t$ equation is based on the fluid dynamic criterion that the nasal airflow is concentrated in the centre of a hypothetical circumference, because most of the energy pushing the air through the nasal cavity is dissipated for effect of the laminar boundary layer flow (LBLF) against the nasal wall for which it is ineffective (Fig. 2).

\section{Results}

No complications were found during nasal manometry. In Table I we report the values of nasal sections expressed in $\mathrm{mm}^{2}$, pressure in mbar and time in sec. We gathered experimental data for a deep breath of a standard-group subject. Observed data were pressure versus (/) time.

The data showed that the effective velocity regarding the

Table I. Values of nasal sections.

\begin{tabular}{|c|c|c|c|c|c|c|c|c|}
\hline Patient no. & Sect $\mathrm{mm}^{2}$ & $\mathrm{mb} / \mathrm{t} 0=0 \mathrm{~s}$ & $\mathrm{mb} / \mathrm{t} 1=0.5$ & $\mathrm{mb} / \mathrm{t} 2=1 \mathrm{~s}$ & $\mathrm{mb} / \mathrm{t} 3=1.5$ & $\mathrm{mb} / \mathrm{t} 4=2.5$ & $\mathrm{mb} / \mathrm{t} 5=3.5$ & $\mathrm{mb} / \mathrm{t} 6=4 \mathrm{~s}$ \\
\hline 1 & 51 & 0 & 1.5 & 3 & 3.7 & 3 & 0.9 & 0 \\
\hline 2 & 51 & 0 & 1.6 & 3.3 & 4 & 2.8 & 0.8 & 0 \\
\hline 3 & 51 & 0 & 1.76 & 3.3 & 3.96 & 3.08 & 0.88 & 0 \\
\hline 4 & 52 & 0 & 1.7 & 3.5 & 3.9 & 3 & 0.9 & 0 \\
\hline 5 & 53 & 0 & 1.9 & 3.1 & 4.2 & 3.2 & 0.6 & 0 \\
\hline 6 & 53 & 0 & 1.7 & 3.5 & 4.2 & 3.1 & 0.7 & 0 \\
\hline 7 & 53 & 0 & 1.8 & 3.4 & 4.3 & 3 & 0.8 & 0 \\
\hline 8 & 54 & 0 & 1.9 & 3.5 & 4.3 & 2.9 & 0.8 & 0 \\
\hline 9 & 54 & 0 & 1.9 & 3.5 & 4 & 3.3 & 0.7 & 0 \\
\hline 10 & 56 & 0 & 2 & 3.7 & 4 & 3.1 & 0.9 & 0 \\
\hline 11 & 56 & 0 & 1.7 & 3.9 & 4.1 & 3.2 & 1 & 0 \\
\hline 12 & 56 & 0 & 1.8 & 4 & 4 & 3.1 & 1.1 & 0 \\
\hline 13 & 57 & 0 & 1.8 & 3.8 & 4.5 & 3.5 & 1.1 & 0 \\
\hline 14 & 57 & 0 & 1.7 & 3.8 & 4.5 & 3.5 & 1.1 & 0 \\
\hline 15 & 57 & 0 & 1.8 & 3.8 & 4.4 & 3.2 & 1.1 & 0 \\
\hline 16 & 58 & 0 & 1.8 & 3.9 & 4.5 & 3.2 & 1.1 & 0 \\
\hline 17 & 58 & 0 & 1.8 & 3.9 & 4.5 & 3.2 & 1.1 & 0 \\
\hline 18 & 58 & 0 & 1.8 & 3.8 & 4.6 & 3.2 & 1.1 & 0 \\
\hline 19 & 59 & 0 & 2.04 & 3.7 & 4.5 & 3.4 & 1 & 0 \\
\hline 20 & 59 & 0 & 2.1 & 3.9 & 4.7 & 3.3 & 1 & 0 \\
\hline 21 & 60 & 0 & 2.07 & 3.89 & 4.67 & 3.63 & 1.03 & 0 \\
\hline 22 & 60 & 0 & 2.06 & 3.8 & 4.7 & 3.53 & 1 & 0 \\
\hline 23 & 61 & 0 & 2 & 3.7 & 4.8 & 3.4 & 0.9 & 0 \\
\hline 24 & 61 & 0 & 1.8 & 3.7 & 4.9 & 3.8 & 0.8 & 0 \\
\hline 25 & 61 & 0 & 2.2 & 3.7 & 4.5 & 3.8 & 0.8 & 0 \\
\hline 26 & 62 & 0 & 1.9 & 4.2 & 4.6 & 3.5 & 1 & 0 \\
\hline 27 & 62 & 0 & 1.9 & 4.1 & 4.9 & 3.5 & 1 & 0 \\
\hline 28 & 63 & 0 & 2 & 4.7 & 4.9 & 3.7 & 0.9 & 0 \\
\hline 29 & 63 & 0 & 2 & 4.6 & 4.8 & 3.8 & 0.89 & 0 \\
\hline 30 & 63 & 0 & 2 & 4.5 & 4.8 & 3.8 & 0.9 & 0 \\
\hline 31 & 64 & 0 & 2.1 & 4 & 4.7 & 3.9 & 1.1 & 0 \\
\hline 32 & 64 & 0 & 2.1 & 4.1 & 4.8 & 3.9 & 1.1 & 0 \\
\hline 33 & 64 & 0 & 2.1 & 4.1 & 4.8 & 3.8 & 1.1 & 0 \\
\hline 34 & 66 & 0 & 2.2 & 4.2 & 4.95 & 3.82 & 1.1 & 0 \\
\hline 35 & 66 & 0 & 2.3 & 4.2 & 4.98 & 3.8 & 1.1 & 0 \\
\hline 36 & 66 & 0 & 2 & 4 & 5.3 & 4.1 & 1 & 0 \\
\hline 37 & 66 & 0 & 1.9 & 4.3 & 5.1 & 3.9 & 1.1 & 0 \\
\hline 38 & 67 & 0 & 2.3 & 4.4 & 5.3 & 4 & 1.1 & 0 \\
\hline 39 & 68 & 0 & 2.32 & 4.3 & 5.4 & 4 & 1 & 0 \\
\hline 40 & 69 & 0 & 2.34 & 4.2 & 5.38 & 4 & 1 & 0 \\
\hline
\end{tabular}

Pat $=$ patient; sect. $=$ section of the nose, $\mathrm{mm}^{2} ;$ mbar. $=$ millibar; $t / \mathrm{s} .=$ time $/$ second . 
calculation of airflow rate is one half the time dependent maximum velocity.

In figure 3 we show the average of the standard group measurements as full circles.

By integrating this curve as a function of time we found that the volume of inhaled air in one nostril $\left(K_{l}\right)$ was 221 $\mathrm{ml}$ and the total volume $\left(K_{2}\right)$ was $442 \mathrm{ml}$.

We compared the volume of inhaled air by the standardgroup subject with an average reference value $(250 \mathrm{ml})$ for each nostril, as reported in the literature ${ }^{7}$.

The standard deviations (SD) for each set of data at a fixed time were then calculated:

$\left[\left(t_{1}\right)=0.20, s\left(t_{2}\right)=0.34, s\left(t_{3}\right)=0.43, s\left(t_{4}\right)=0.36, s\left(t_{5}\right)=0.14\right]$.

These statistical parameters suggested the presence of two limiting curves, one below and one above the average representative curve depicted in Figure 1, enclosing an area into which the respiratory pattern of healthy people falls. Therefore, two limiting values of the volume of inhaled air per nostril exist: $K_{\text {inf }}=191 \mathrm{ml}$ and $K_{\text {sup }}=267 \mathrm{ml}$.

\section{Discussion}

Nasal surgery aims at improving nasal breathing, yet decisions pertaining to the need for surgical treatment implies a significant degree of subjective judgement by the surgeon ${ }^{13}$. So far, NMR and AR are the most widely used tools to evaluate the nasal airflow, but they provide only a global airflow measurement, without temporal or spatial details. Furthermore, these methods do not suffice to reliably guide the surgeon in the choice of the best therapeutic strategy ${ }^{14}$. In addition, they do not provide information about local details of nasal airflow, often of utmost importance from a clinical standpoint. Although $\mathrm{AR}^{10}$ produces a more detailed evaluation of the geometry of the nasal cavities, it does not evaluate the flow field, but only the geometric boundaries. Indeed, previous studies ${ }^{15}$ showed the validity of AR in the evaluation of functional outcomes following endoscopic surgery in correlation with volumetric $\mathrm{CT}$, but it did not provide any information on the physiology of nasal pressures ${ }^{16}$.

Unlike NMR or AR, CFD showing the airflow characteristics in 3D can make accurate predictions about the variables of fluids in a computer model ${ }^{17}$. For instance, many authors made airflow calculations by reconstructing the nasal geometry from CT sequences of healthy subjects, whose nose was assumed to be normal in terms of flow and symmetry ${ }^{18}$. However, they pointed out some challenging aspects related to the geometric complexity of nasal airways: the non-uniqueness of standard measurement of engineering type procedures applied to humans, the presence of physical phenomena implicated (viscosity, flow conditions, Reynolds number, wall roughness, heat transfer, humidity, fluid tissue interaction, turbulence) and, most important, the fact that CFD studies are only

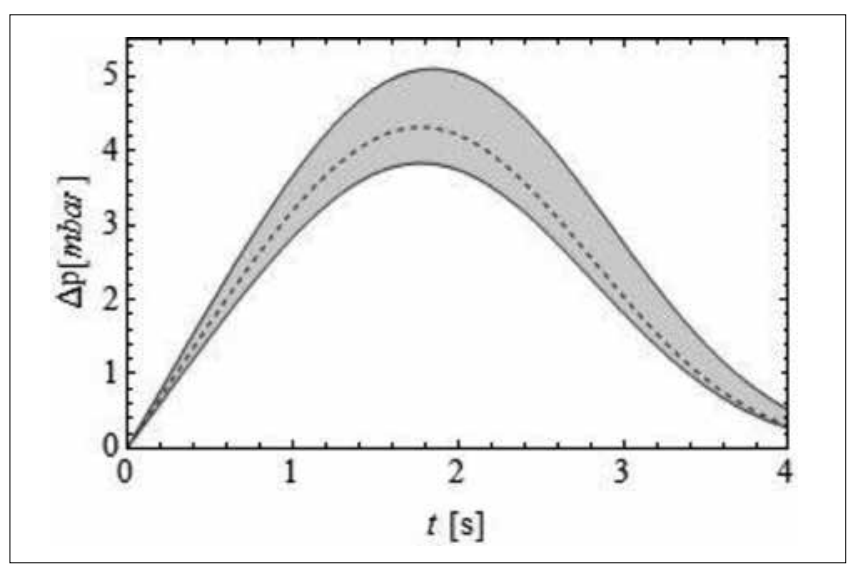

Fig. 3. Fitting experimental data of gauge pressure in the nasal valve by means of the continuous function $\Delta p(t)=A$ t $\exp (-B$ t3) with $A=3.3914$ and $B=0.05951$, we depicted an area into which the air respiration patterns of healthy people fall.

simulations and the predicted results derived from complex calculations of the Navier-Stokes equation, which may not represent real-life conditions ${ }^{10} 18$.

In our study, we evaluated the nasal airflow by means of a mathematical model based on the validity of Bernoulli's equation in the central part of the nostril. To take account of nasal airflow viscosity, we applied a quadratic velocity profile in the nostril, whose section was assumed to be circular for simplicity. Under these assumptions experimental data, reporting the gauge pressure $\Delta p_{N}$ inside the nostril as a function of time, were gathered by DDM-MG ${ }_{1}$ Endoflower that allowed to study the pressure inside the nose, pointing the probe in the specific nasal subsite we wanted to investigate ${ }^{1920}$.

The inner nasal structures were assimilated to a sequence of tubes under stationary conditions in which single particles close to the centre of the flow described laminar trajectories without energy loss ${ }^{21}$. Although this model represents a valid approximation for airflow away from the ducts, a viscous effect could be considered in the proximity of the walls of the nasal cavity. Therefore, in our model we assumed the validity of Bernoulli's equation in the central part of the duct, where the gradient of the velocity profile could be considered to be negligible. To achieve mathematical computed values of the volume of air during a single inhalation act, obtained pressure values were processed with data from the corresponding CT scan slide.

We found that the average inhaled air volume in a single nostril in a standard-group subject during a deep breath was $221 \mathrm{ml}$, with an overall volume of $442 \mathrm{ml}$. Conversely, the literature reports values of $250 \mathrm{ml}$ and $500 \mathrm{ml}$, respectively ${ }^{18}$.

Our study, for the first time, identified two limiting curves, one below and one above an average representative curve, describing the time dependence of the gauge pressure inside a single nostril. These upper and lower curves enclosed an area into which the airflow pattern of healthy people falls. Two limiting values of the volume of inhaled 
air per nostril, $K_{i n f}=191 \mathrm{ml}$ and $K_{\text {sup }}=267 \mathrm{ml}$, were found. It can be argued that in order to measure nasal airflow a CT scan is needed and this would limit the clinical usefulness of the model. However, CT is not strictly necessary as the probe is placed under endoscopic guidance, which allows the perfect positioning of the probe at the chosen subsite. It is also to be considered that CT is often performed in any case as a diagnostic and preoperative tool for surgical planning.

\section{Conclusions}

In conclusion, despite recent advances in nasal diagnostics, there are still open questions on the management of nasal obstruction and on the opportunity to perform nasal surgery 2223 . Furthermore, given the lack of reliable tools to objectively measure nasal resistances and to identify the right subsite of nasal obstruction, the goal of this study was to allow clinicians to obtain pressure measurements over time at various subsites of nasal airway to identify which ones are obstructed. In the present study, a mathematical model has been applied to healthy subjects to assess the normal range of resistances, with the aim to compare, in future studies, these data with those from subjects with nasal obstruction at different subsites ${ }^{18}$.

\section{Acknowledgments}

We are also deeply indebted to Anna Marino, MD, for her cooperation.

\section{References}

1 Zhao K, Blacker K, Luo Y, et al. Perceiving nasal patency through mucosal cooling rather than air temperature or nasal resistance. PLoS ONE 2011;6: e24618.

2 Hong SD, Lee NJ, Cho HJ, et al. Predictive factors of subjective outcomes after septoplasty with and without turbinoplasty: can individual perceptual differences of the air passage be a main factor? Int Forum Allergy Rhinol 2015;5:616-21.

3 Zojaji R, Keshavarzmanesh M, Bakhshaee M, et al. The effects of inferior turbinoplasty on nasal airflow during cosmetic rhinoplasty. Acta Otorhinolaryngol Ital 2016;36:97-100.

4 Yu S, Liu Y, Sun X, et al. Influence of nasal structure on the distribution of airflow in nasal cavity. Rhinology 2008;46:137-43.

5 Eichhorn KW, Schneider B, Bley TA, et al. CT rhinometry: a correlation of rhinomanometry and multiplanar computer tomography of the nasal cavity. HNO 2012;60:1067-74.

6 Gamerra M, De Luca R, Pagano G, et al. The nose and sinus manometry: a bio-physical model applied to functional endoscopic sinus surgery. J Biol Regul Homeost Agents 2013;27:1021-7.
7 Huizing EH, De Groot Johan AM. Functional Reconstructive Nasal Surgery. Stuttgard: Thieme; 2003.

8 Cantone E, Castagna G, Ferranti I, et al. Concha bullosa related headache disability. Eur Rev Med Pharmacol Sci 2015;19:2327-30.

9 Clement PA, Halewyck S, Gordts F, et al. Critical evaluation of different objective techniques of nasal airway assessment: a clinical review. Eur Arch Otorhinolaryngol 2014;271:2617-25.

10 Wang de Y, Lee HP, Gordon BR. Impacts of fluid dynamics simulation in study of nasal airflow physiology and pathophysiology in realistic human three-dimensional nose models. Clin Exp Otorhinolaryngol 2012;5:181-87.

11 Sung Kyun K. Yang N, Jee-In K, et al. Patient specific CFD models of nasal airflow: overview of methods and challenges. J Biomech 2013;46:299-306.

12 Tan J, Han D, Wang J, et al. Numerical simulation of normal nasal cavity airflow in Chinese adult: a computational flow $d y$ namics model. Eur Arch Otorhinolaryngol 2012;269:881-9.

13 Cantone E, Castagna G, Sicignano S, et al. Impact of intranasal sodium hyaluronate on the short-term quality of life of patients undergoing functional endoscopic sinus surgery for chronic rhinosinusitis. Int Forum Allergy Rhinol 2014;4:484-7.

14 Ting L, Demin H, Jie W, et al. Effects of septal deviation on the airflow characteristics: using computational fluid $d y$ namics models. Acta Oto-Laryngologica 2012;132:290-8.

15 Yang N, Kang Soo C, Seung-Kyu C, et al. Effects of single-sided inferior turbinectomy on nasal function and airflow characteristics. Respir Physiol Neurobiol 2012;180:289-97.

16 Kass ES, Salman S, Montgomery WW. Manometric study of complete ostial occlusion in chronic maxillary atelectasis. Laryngoscope 1996;106:1255-8.

17 Wide K, Sipila J, Suonpaa J. The value of computerised rhinomanometry and a simple manometry with saline in predicting the outcome of patients with acute trephined frontal sinusitis. Rhinology 1996;34:151-5.

18 Anthony TR, Anderson KR. Computational fluid dynamics investigation of human aspiration in low-velocity air: orientation effects on mouth-breathing simulations. Ann Occup Hyg 2013;57:740-57.

19 Gamerra M, Bruno R, Pagano G, et al. The nose function and aesthetic. Ann Otorhinolaringology Iber-Amer 2004;31:307-23.

20 Bertrand B, Collet S, Betsch C, et al. Diagnostic techniques in chronic sinusitis: endoscopy, sinusomanometry. Acta Otorhinolaryngol Belg 1997;51:259-69.

21 De Luca R, Gamerra M, Sorrentino G, et al. Nose and sinus air flow model. Natural Science 2014; 6:685-90.

22 De Corso E, Bastanza G, Di Donfrancesco V, et al. Radiofrequency volumetric inferior turbinate reduction: long-term clinical results. Acta Otorhinolaryngol Ital 2016;36:199-205.

23 Zojaji R, Keshavarzmanesh M, Bakhshaee M, et al. The effects of inferior turbinoplasty on nasal airflow during cosmetic rhinoplasty. Acta Otorhinolaryngol Ital 2016;36:97-100.

Address for correspondence: Elena Cantone, Department of Neuroscience, ENT Unit, University of Naples "Federico II", via Pansini, 580100 Naples, Italy. Tel. +39 081 7463598. Fax +39081 7463592. E-mail: elena.cantone@unina.it 